Cartwright, T. N., Worrell, J. C. , Marchetti, L., Dowling, C. M., Knox, A., Kiely, P., Mann, J., Mann, D. A. and Wilson, C. L. (2018) HDAC1 interacts with the p50 NF- $\kappa \mathrm{B}$ subunit via its nuclear localization sequence to constrain inflammatory gene expression. Biochimica et Biophysica Acta: Gene Regulatory Mechanisms, 1861(10), pp. 962-970. (doi:10.1016/j.bbagrm.2018.09.001)

There may be differences between this version and the published version. You are advised to consult the publisher's version if you wish to cite from it.

\title{
http://eprints.gla.ac.uk/196544/
}

Deposited on: 17 September 2019

Enlighten - Research publications by members of the University of Glasgow

http://eprints.gla.ac.uk 


\section{HDAC1 Interacts with the p50 NF-кB Subunit via its Nuclear Localization Sequence to Constrain Inflammatory Gene Expression}

Tyrell N. Cartwright ${ }^{1}$, Julie C. Worrell ${ }^{1}$, Letizia Marchetti ${ }^{1}$, Catríona M.Dowling ${ }^{2}$, Amber Knox ${ }^{1}$, Patrick Kiely ${ }^{2}$, Jelena Mann ${ }^{1}$, Derek A. Mann ${ }^{1}$ and Caroline L. Wilson ${ }^{1 *}$.

${ }^{1}$ Newcastle Fibrosis Research Group, Institute of Cellular Medicine, Newcastle University

${ }^{2}$ Health Research Institute, University of Limerick

* Corresponding Author 


\begin{abstract}
The NF- $\kappa$ B p50 subunit is an important regulator of inflammation, with recent experimental evidence to support it also having a tumor suppressor role. Classically, p50 functions in heterodimeric form with the RelA (p65) NF- $\kappa \mathrm{B}$ subunit to activate inflammatory genes. However, p50 also forms homodimers which actively repress NF- $\kappa \mathrm{B}$-dependent inflammatory gene expression and exert an important brake on the inflammatory process. This repressive activity of p50:p50 is thought to be in part mediated by an interaction with the epigenetic repressor protein Histone Deacetylase 1 (HDAC1). However, neither the interaction of p50 with HDAC1 nor the requirement of HDAC1 for the repressive activities of p50 have been well defined. Here we employed in silico prediction with in vitro assays to map sites of interaction of HDAC1 on the p50 protein. Directed mutagenesis of one such region resulted in almost complete loss of HDAC1 binding to p50. Transfected mutant p50 protein lacking the putative HDAC1 docking motif resulted in enhanced cytokine and chemokine expression when compared with cells expressing a transfected wild type p50. In addition, expression of this mutant p50 was associated with enhanced chemoattraction of neutrophils and acetylation of known inflammatory genes demonstrating the likely importance of the p50:HDAC1 interaction for controlling inflammation. These new insights provide an advance on current knowledge of the mechanisms

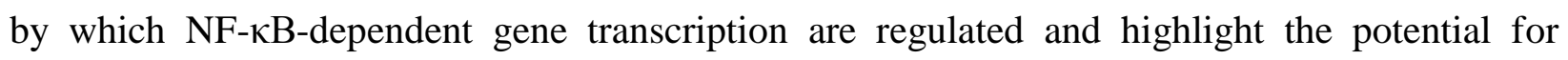
manipulation of p50:HDAC1 interactions to bring about experimental modulation of chronic inflammation and pathologies associated with dysregulated neutrophil accumulation and activation.
\end{abstract}

Keywords: NF-кB, HDAC1, p50, Inflammation, Neutrophil. 


\section{Introduction}

NF- $\kappa \mathrm{B}$ is a family of five transcription factors (RelA (p65), RelB, c-Rel, p100/p52 p105/p50), that combine to control expression of hundreds of genes that impact on the regulation of cell proliferation and survival, differentiation, inflammation, metabolism, tissue repair and regeneration, fibrosis and cancer [1]. The most well-known and functionally characterized form of NF- $\mathrm{NB}$ is the so-called canonical RelA:p50 heterodimer that is activated in response to a variety of extracellular inflammatory stimuli (e.g. TNF, IL-1 and LPS). RelA:p50 is required for mounting inflammatory responses through RelA-mediated transcriptional activation of key cytokine and chemokine genes. Given the potential for inflammation to cause tissue damage, the activities of RelA are tightly regulated by a variety of inhibitory checkpoints and negative feedback mechanisms [2]. A major focus for our laboratory has been the functions of the p50 $\mathrm{NF}-\kappa \mathrm{B}$ subunit expressed from the NFKB1 gene and in particular its distinct role when operating without RelA as a dimer partner [3]. Expression of p50 is found in most cell types under resting conditions, however the subunit can be induced to higher levels of expression in response to inflammatory stimuli [4]. Although p50 is generated directly from NFKB1 transcripts [5], a predominant source in inflammatory states is via processing of its p105 precursor. This processing involves signal-induced, ubiquitin-dependent limited proteolysis regulated by the KPC1 ubiquitin E3 ligase [6]. An important structural feature of p50 is its lack of the transactivation domain typically found in the Rel proteins, as such it relies upon its Rel dimerization partners (RelA, c-Rel and RelB) to induce gene expression. Importantly, p50 can also form homodimers, their assembly being under distinct post-translational control with evidence for the requirement of specific serine residues (e.g. Ser-65, Ser-337 and Ser-340) which appear to be non-essential for heterodimerisation [3,7]. These observations raise the potential for selective modulation of NF- $\kappa \mathrm{B}$ activities that are under the control of p50:p50 dimers.

In striking contrast to embryonic lethality found in rela ${ }^{-/-}$mice, the absence of p50 in $n f k b 1^{-/}$ mice results in loss of immune tolerance, heightened inflammatory responses to tissue damage and spontaneous inflammatory-driven ageing, these phenotypes collectively indicating that p50 exerts important anti-inflammatory control in tissues [8]. We recently confirmed by a genetic approach that these anti-inflammatory effects are a consequence of activities of the p50:p50 homodimer. Mice genetically engineered to be selectively incapable of the assembly of 
homodimers phenocopied many of the pro-inflammatory features of $n f k b 1^{-1}$ mice [3]. The precise mechanism by which homodimers suppress inflammation remains poorly defined, but may involve direct competition with Rel-containing dimers for occupancy of $\kappa \mathrm{B}$ DNA binding motifs or a more active process in which recruitment of histone deacetylases promotes local chromatin remodeling into a repressed state [9]. In both cases, the interaction of the homodimer with co-repressors is an important regulatory feature. Bcl3 is an IkB-like protein that has been reported to directly physically combine with p50:p50 dimers to prevent ubiquitin-mediated degradation and thereby enhances the stability of homodimers bound at $\mathrm{\kappa B}$ DNA binding motifs [10]. HDAC1 is a class I nuclear histone deacetylase that is also proposed to physically complex with p50 homodimers and to mediate repressive histone deacetylation of local chromatin at NF$\kappa \mathrm{B}$-regulated genes. It is additionally suggested that CCAAT enhancer-binding proteins (C/EBP) may selectively remove HDAC1 from homodimers resulting in release of a subset of NF- $\mathrm{kB}$ target genes from transcriptional repression [11]. However, the precise nature of the interaction of the HDACs with p50 is poorly defined and the degree to which HDAC:p50 interactions contribute to the control of transcription is unclear.

Here, we define a specific site of interaction of HDAC1 on the p50 protein and report that mutation of this HDAC1 binding motif results in modulation of histone acetylation at a subset of $\mathrm{NF}-\mathrm{\kappa B}$ target genes and is associated with elevated chemokine expression and enhanced neutrophil chemotaxis. These discoveries significantly advance our understanding of a transcriptional repression mechanism that acts as a physiologically important constraint on NFкB-dependent inflammation. 


\section{Materials and Methods}

\subsection{Cell Culture}

Cos-7, U2OS, HELA and $n f k b 1^{-/-}$Murine Embryonic Fibroblasts (MEFs) were maintained in DMEM (GIBCO) supplemented with $100 \mathrm{U} / \mathrm{ml}$ penicillin, $100 \mu \mathrm{g} / \mathrm{ml}$ streptomycin, $2 \mathrm{mM}$ L-glutamine and $10 \%$ fetal bovine serum at $37^{\circ} \mathrm{C}$ at an atmosphere of $5 \% \mathrm{CO}_{2}$

\subsection{Generation of Mutant p50-FLAG Constructs}

Mutant constructs were all generated from a parent p50-FLAG pcDNA3.1 backbone kindly provided by N. D. Perkins (Newcastle University). Mutations were generated using a Q5 Site-Directed Mutagenesis Kit (E0554S, New England Biolabs). Briefly, template p50-FLAG pcDNA3.1 was incubated $95^{\circ} \mathrm{C}$ followed by 3 minute cycles at the appropriate annealing temperate with a final incubation at $72^{\circ} \mathrm{C}$ for 10 minutes. This linear mutant DNA was then circularized using the KDL enzyme mix at room temperature for 15 minutes and products transformed into DH5 $\alpha$ competent E.Coli (C2987H, New England Biolabs) and colonies selected by antibiotic resistance. Plasmids were then purified using an Endotoxin Free Plasmid Maxi Kit (12163, Qiagen). All mutants were confirmed by Sanger sequencing (GATC Bio-tech). Primers used in mutagenesis can be found in Table 1.

\subsection{Transfection, Co-Transfection and Luciferase Reporter Assay}

Generated mammalian expression constructs were transfected into Cos-7, U2OS, HELA and $n f k b 1^{-/-}$MEF cell lines for 48 hours (ChIP Assays), 24 hours (Nuclear localization, Luciferase Assay, Endogenous and FLAG Co-IP) or 18 hours (followed by TNF $\alpha$ treatment for 6 hours at 10ng/ml) using Effectene Transfection reagent (301427, Qiagen) following manufacturer's instructions. Briefly, cells were seeded to 40-60\% confluency and DNA for transfection incubated in EC Buffer with Enhancer and Effectene reagent for 5 and 10 minutes respectively. This mixture was further diluted in DMEM and added to cells under normal culture conditions for the indicated time period. Following incubation cells were then harvested for Western Blot, Precipitation assays or Luciferase assay. Luciferase assays were performed using a Dual Luciferase Assay Reporter System (E1910, Promega) following manufacturer's instructions. 
Relative luminescence was normalized to co-transfected Renilla luciferase, control luciferase reporter as well as total protein. Transfection DNA concentrations can be found in Table 2.

\subsection{Endogenous Co-Immunoprecipitation and FLAG Immunoprecipitation Assay}

Tissue or cells were washed in PBS and lysed in Co-IP lysis buffer (50mM Tris-HCL pH 7.4, $150 \mathrm{mM} \mathrm{NaCl}, 1 \mathrm{mM}$ EDTA and 1\% Triton-X) with fresh protease inhibitors (118735801 Roche).

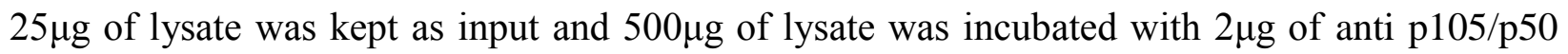
(D7H5M CST), nuclear localization specific anti p50/p105 (sc-114x Santa Cruz), anti-p65 (sc 372 Santa Cruz), anti IкB $\alpha$ (L35AS CST), normal IgG (ab46540-1 Abcam) or anti-FLAG beads (A2220 Sigma) for 1 hour at $4^{\circ} \mathrm{C}$ diluted to a total volume of 500 $\mu 1$ in Wash Buffer (50mM TrisHCL pH 7.4 and 150mM NaCl). Apart from samples with FLAG beads, a 1:1 mix of Protein A and Protein G agarose beads (P6649 and P3296 Sigma) were then added to samples and incubated a further hour at $4^{\circ} \mathrm{C}$. All samples were then washed in Wash Buffer and bound complexes eluted by boiling with $1 \mathrm{X}$ SDS-Loading Dye at $95^{\circ} \mathrm{C}$ or by incubation with Wash Buffer containing excess FLAG peptide in the case of FLAG IPs. Samples along with inputs were then assessed by Western Blot.

\subsection{Western Blot}

Total cells lysates or immunoprecipitated samples were quantified by DC protein assay (BioRad) and equal amounts fractionated by $8 \%$ SDS-PAGE, transferred to nitrocellulose membrane and blocked in Tris-buffered saline and Tween $20(0.1 \%)$ containing 5\% milk protein before overnight incubation (1 hour for HRP conjugates) with primary antibodies to (1:1000) p65 (sc372 Santa Cruz), (1:2000) GAPDH (ab22555 Abcam), (1:1000) nfkb1-HRP conjugate (ab195854 Abcam) or (1:1000) HDAC1-HRP conjugate (ab193096 Abcam). Next, membranes were washed in TBS-T and further incubated with (1:1000) HRP-conjugated mouse anti-rabbit IgG (7074S CST). After a final wash antigens were detected by chemiluminesence (Amersham Biosciences).

\subsection{Nuclear and Cytoplasmic Extracts}

$8 \times 10^{5}$ U2OS, $6 \times 10^{5} n f k b 1^{-/-}$or $6 \times 10^{5}$ WT MEF cells were transfected with either $200 \mathrm{ng} / \mathrm{ml}$ or $50 \mathrm{ng} / \mathrm{ml} \mathrm{WT}, \mathrm{p} 50^{\mathrm{Mu}}$ or vehicle. Cells were treated with $10 \mathrm{ng} / \mathrm{ml} \mathrm{TNFa}$ for 6 hours or media alone and subsequently washed with PBS and cell pellets lysed in cytoplasmic lysis buffer (50mM 
Tris-HCL pH 7.4, 50mM NaCl, 1mM EDTA, protease inhibitors and $0.1 \%$ Triton-X) and homogenized using a 27G needle. Supernatant was kept as cytoplasmic extract and pelleted nuclei were washed in cytoplasmic lysis buffer and resuspended in RIPA lysis buffer. Samples were then analyzed by western blot.

\subsection{Immunofluorescence Microscopy}

U2OS cells were seeded at 40-50\% confluency in 4 well chamber slides and transfected with $250 \mathrm{ng} / \mathrm{ml}$ of either WT p50-FLAG, p50 ${ }^{\mathrm{Mu}}$-FLAG or vehicle only and incubated for 24 hours. Cells were then washed with PBS and fixed in $4 \%$ formaldehyde followed by permeabilization with $0.1 \%$ Triton-X and blocked for 1 hour in 5\% Bovine Serum Albumin(BSA). Cells were then incubated over night at $4^{\circ}$ with anti-FLAG-FITC antibody (F4049, Sigma) in 5\% BSA. Cells were then washed in PBS and mounted with ProLong Gold Antifade Mountant with DAPI (P36935, Thermo-Fisher) and imaged on a Nikon upright Confocal Microscope at x200.

\subsection{Enzyme Linked Immunosorbent Assay}

Enzyme Linked Immunosorbent Assay Mouse IL-6 DuoSet (DY406, R\&D Systems) was used to quantify total mouse IL-6 in supernatant of $n f k b 1^{-/-}$MEFs transfected with Vehicle, WT or p50 $0^{\mathrm{Mu}}$ for 18 hours followed by treatment with or without TNF $\alpha(10 \mathrm{ng} / \mathrm{ml})$ for 6 hours. This sandwich ELISA was performed as per manufacture instruction.

\subsection{Chromatin Immunoprecipitation (ChIP)}

Briefly, $n f k b 1^{-/-}$MEFs transfected for 48 hours with $200 \mathrm{ng} / \mathrm{ml}$ WT p50, p50 $0^{\mathrm{Mu}}$ or vehicle only were cross-linked by addition of formalin at $1 \%$ final concentration for 5 minutes at RT, then quenched with glycine buffer at final concentration of $125 \mathrm{mM}$. The cells were rinsed in cold PBS, and harvested into PBS with protease inhibitors and centrifuged for $5 \mathrm{~min}, 4^{\circ} \mathrm{C}, 1,000 \mathrm{x}$. The pellet was resuspended in ChIP lysis buffer (1\% SDS, 10mM EDTA, 50mM Tris-HCl $\mathrm{pH} 8.1$ ) and incubated for $20 \mathrm{~min}$ on ice. Lysate was sonicated to generate average size fragments of 200-700bp; cell debris was pelleted by centrifugation for $10 \mathrm{~min}, 4^{\circ} \mathrm{C}, 8,000 \mathrm{x} \mathrm{g} .25 \mu \mathrm{g}$ of chromatin per IP was diluted 1:10 with dilution buffer (1.1\% Triton, 1.2mM EDTA, 16.7mM TRIS-HCl pH8.1,167mM NaCl, with protease inhibitors) and precleared by incubating with 25 $\mu l$ blocked Staph A membranes (Sigma, P7155) for 15 minutes. Pre-cleared chromatin was then incubated with $5 \mu \mathrm{g}$ anti acetyl H3 (ab47915, Abcam), anti p50/p105 (ab7971, Abcam) or 
irrelevant IgG (ab171870, Abcam) overnight at $4^{\circ} \mathrm{C}$. Immunoprecipitated chromatin was collected with blocked Staph A membranes, which were then sequentially washed with cold buffers; Low Salt Wash Buffer (0.1\% SDS, 1\% Triton X-100, 2 mM EDTA, 20 mM Tris-HCl pH 8.0, 150 mM NaCl), High Salt Wash Buffer (0.1\% SDS, 1\% Triton X-100, 2 mM EDTA, 20 $\mathrm{mM}$ Tris- $\mathrm{HCl} \mathrm{pH}$ 8.0, $500 \mathrm{mM} \mathrm{NaCl}$ ), LiCl Wash Buffer (0.25 M LiCl, 1\% NP-40, 1\% Sodium Deoxycholate, $1 \mathrm{mM}$ EDTA, $10 \mathrm{mM}$ Tris- $\mathrm{HCl} \mathrm{pH}$ 8.0) and two washes in TE Buffer (10 mM Tris $\mathrm{pH} 8.0,1 \mathrm{mM}$ EDTA). The immunoprecipitated chromatin was eluted in $500 \mu$ l Elution buffer (1\% SDS, 100mM NaHCO3), cross-links reversed and DNA obtained by phenol:chloroform extraction and gel purification. The level of $\mathrm{Cxcl} 1$ and $\mathrm{Cxcl} 2$ gene promoter association with acetylated H3 were quantitatively measured by real-time PCR using primers listed Table 1.

\subsection{RNA Isolation and Quantitative Real Time PCR}

Total RNA was isolated from cells using an RNeasy Mini Kit (74104, Qiagen) and treated with DNAse. This RNA was then used as a template for the synthesis of complementary DNA using random primers (Promega). SYBR Green quantitative RT-PCR was performed using primers listed in Table 1.

\subsection{Neutrophil Isolation and Chemotaxis Assay}

Bone marrow derived neutrophils were isolated from the femur and tibia of C57BL/6 WT mice by flushing HBSS supplemented with 20mM HEPES and 0.5\% fetal calf serum. Red blood cells were lysed with BD PharmLyse buffer (BD Biosciences; Ref: 555899) and cellular debris removed using a $40 \mu \mathrm{m}$ filter. Neutrophils were isolated by negative depletion of non-target cells through magnetic-labelling and column separation (Neutrophil Isolation Kit - Mouse, Miltenyi

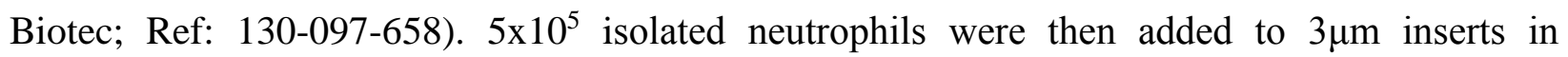
complete DMEM which were inserted into wells containing media from $n f k b 1^{-/-}$MEFs reconstituted with WT p50, p50 $\mathrm{Mu}$, vehicle controls or unconditioned media only with or without TNF $\alpha$. After 2 hours neutrophils that migrated into the lower chamber were stained with trypan blue and counted by hemocytometer. Chemotaxis was performed in triplicate with $\mathrm{n}=3$ conditioned media. 


\subsection{2 p50 Peptide and Substitution Array}

p50 peptide and alanine substitution arrays were kindly provided by the Kiely Lab and were generated as previously described [12]. Membranes were blocked in 5\% TBS-T for one hour followed by incubation with anti (1:5000) 6x-His Tag Monoclonal Antibody HRP (R931-25, Invitrogen) for 2 hours at room temperature and developed by chemiluminescence (Amersham Biosciences) to assess peptide antibody cross reactivity. 6xHis tagged recombinant HDAC1 (14838 , Millipore) at $5 \mu \mathrm{g} / \mathrm{ml}$ in $1 \%$ milk TBS-T was then added over night at $4^{\circ} \mathrm{C}$ followed by TBS$\mathrm{T}$ wash and incubation again with antibody and detection as described. This procedure was also performed on the p50 alanine substitution array. Images are representative of two independent arrays.

\subsection{GRAMM-X Protein Interaction Prediction and Visualization}

p50 HDAC1 interaction predictions were performed by the GRAMM-X software for ridged body protein-protein interactions. Chain $\mathrm{A}$ and $\mathrm{B}$ of the available crystal structure of the p50 homodimer (PDB ID:1NFK [13]) were used as a receptor molecule for its ligand, chain B of the HDAC1 crystal structure (PDB ID:4BKX [14]). Potential interacting intermolecular hydrogen bonds were calculated using the HBond function in UCSC Chimera with relaxed bond constraints of $7 \AA$ and $20 \%$ bond angle flexibility. Structural modelling and images were generated in UCSC Chimera [15].

\subsection{Transfection Efficiency}

$2 \times 10^{4} n f k b 1^{-1}$ MEF's were seeded in 4 well chamber slides and transfected with $200 \mathrm{ng} / \mathrm{ml}$ pcDNA3 GFP and imaged after 24 hours at x 100 magnification. pcDNA3 EGFP was a gift from Doug Golenbock (Addgene plasmid \# 13031).

\subsection{Statistical Analysis}

Data are expressed as mean \pm S.E.M. Microsoft Excel was used to perform unpaired Students $t$ test and one way A.N.O.V.A.s and $* P<0.05$, $* * P<0.01$ or $* * * P<0.001$ was considered significant. 


\section{Results and Discussion}

\section{1 p50 Interacts with HDAC1 in primary and transformed cells.}

Previous in vitro biochemical investigations in mouse leukemia cell lines demonstrated potential for a p50:HDAC1 interaction [16]. However, as it has yet to be demonstrated in vivo, we established co-immunoprecipitation (CoIP) assays to detect p50:HDAC1 interactions from primary cells. As shown in Fig 1A, under resting conditions in mouse liver tissue we were able to CoIP HDAC1 with an anti-p50/p105 antibody indicating an interaction does occur between the two proteins in primary tissue. We also confirmed detection of the interaction in human Hela cells and demonstrated that stimulation with TNF $\alpha$ led to reduced CoIP of HDAC1 (Supp. 1A). We conclude that a p50:HDAC1 complex can be detected in primary and cell lines and may be modulated in response to inflammatory stimuli, the latter in keeping with a model in which stimulated release of HDAC1 from p50 may serve to relieve HDAC1-mediated transcriptional repression at NF- $\kappa \mathrm{B}$ target genes [11]. It should be noted this suggests that $\mathrm{TNF} \alpha$ does not disrupt the p50:HDAC1 interaction directly, however, it is thought that it may allow the release of p65 and shifts the composition of dimers from a repressive p50:p50:HDAC1 complex to that of a p65:p50 heterodimer which can then recruit co-factors including histone acetyl transferases, to upregulate gene transcription.

We next employed in silico modelling to determine potential sites of interaction of HDAC1 on the $\mathrm{p} 50$ protein in the context of the structure determined from its DNA-bound homodimer form. Using the GRAMM-X server for rigid body interactions we determined the highest scoring possible orientations for predicted binding of HDAC1, taking into account molecular symmetry and potential DNA clashes (Supp. 1B) [17]. This analysis identified two physically distinct sites of predicted HDAC1 interaction on p50 that are shown in Fig 1B. An important caveat of this analysis is that calculations were restricted by the incomplete resolved crystal structure of both proteins (p50 PDB: 1NFK [13], HDAC1 PDB: 4BKX [14]). However, this prediction still provides valuable information as it pertains to the broad regions of affinity between the two molecules and their suitability for direct interaction. To biochemically test this in silico data, we employed an array of 18 amino acid long peptides that overlap and span the full length of p50 to determine regions of the protein with the potential to directly bind to recombinant HDAC1. 
Peptides shown under UV (Supp. 1C) were first probed for non-specific binding using detection antibody (Supp. D) before being incubated with and probed for HDAC1 binding. Four major regions of interaction were revealed and are identified by distinct color shading in the primary sequence format in Fig 1C and in a structural format in Fig 1D. Importantly, these peptide interactions confirmed the two predicted interaction sites on p50 determined by the in silico analysis. We therefore conclude that HDAC1 may complex with p50 via physical interactions at two distinct regions of the NF- $\mathrm{kB}$ subunit. One of these two sites, located at the far c-terminal region of p50 (shaded in yellow) particularly attracted our attention as it showed consistent binding with HDAC1 across multiple peptides (Fig 1C), matched the highest scoring for interaction in the in silico analysis and has previously been reported as a site of interaction of p50 with IкB $\alpha$ and $\mathrm{Bcl} 3[18,19]$. Additionally, the other potential site (blue) highlighted residues with poorer accessibility which reside near the DNA binding domain of p50. 

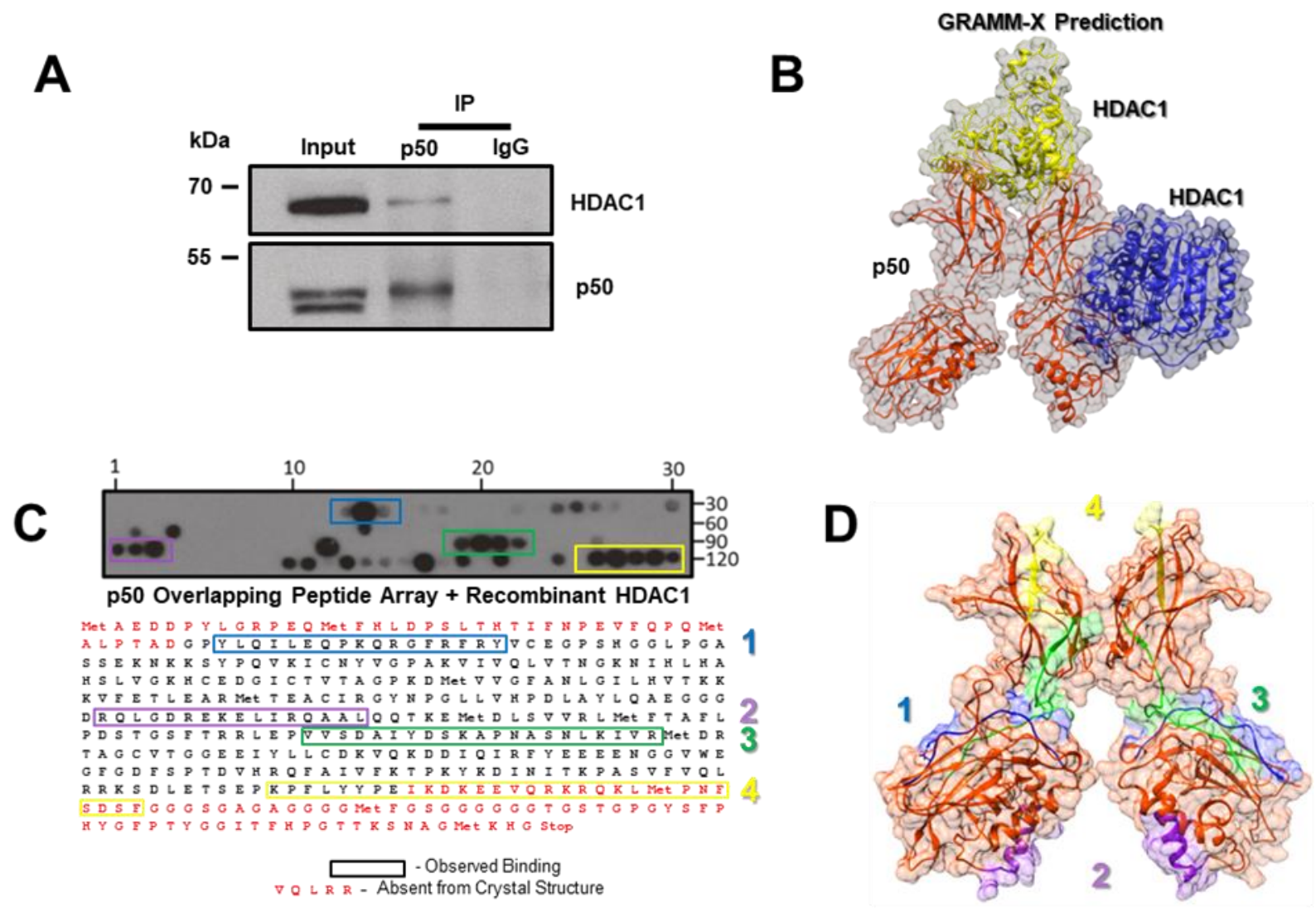

Figure 1. p50 Associates with HDAC1 in vivo

A. Western Blot analysis of an endogenous co-immunoprecipitation showing HDACl precipitates in complex with p50 in untreated three-month-old WT whole liver extracts. Detection antibody produces a lower molecular weight nonspecific band in whole mouse tissue. Representative image of three independent experiments. (IP antibody: anti-p105/p50 (D7H5M CST), Detection antibodies: anti p50HRP [ab195854 Abcam] anti-HDAC1-HRP conjugate [ab193096 Abcam]. B. Predicted association of HDAC1 [Yellow \& Blue, PDB ID: 4BKX] with a p50 homodimer [Orange, PDB ID: 1NFK] depicting two potential sites of interaction as solved by the GRAMM-X software for ridged body protein docking. $C$. Peptide array of 120 sequential peptides of 18 amino acids spanning the length of p50 probed with recombinant HDAC1-Hisx3 highlighting multiple sights of potential interaction (n=2 (anti-6x-His Tag Monoclonal Antibody HRP, R931-25, Invitrogen). D. All regions of potential interaction discovered in the peptide array mapped to the crystal structure of the p50 homodimer. 


\subsection{HDAC1 interacts with p50 at its nuclear localization signal.}

To better define the c-terminal binding site we employed scanning alanine substitutions into the peptides derived from this region that were previously shown to directly bind to recombinant HDAC1 and asked how these mutations affected the interaction. Fig $2 \mathbf{A}$ shows the resulting raw data which was used to locate an 7 amino acid long motif VQRKRQK (aa's 359-365 of Human p50) which showed differential binding. Fig 2B illustrated the degree to which each residue contributes to this apparent loss of binding specifically in peptide 120 . When mutated to alanine, residues in this motif reduce the ability of recombinant HDAC1 to bind with peptides as it had bound previously. On inspection we realized that the VQRKRQK motif lies within the p50 nuclear localization signal (NLS) sequence at the far c-terminal of p50 and that this region is absent from the published protein crystal structure (Fig 2C). To validate that this motif is required for HDAC1:p50 binding in vivo we employed site-directed mutagenesis to generate a series of mutant c-terminal FLAG-tagged p50 cDNA expression constructs carrying one or more amino acid switches to alanine within the VQRKRQK region. WT and mutant p50-FLAG cDNAs together with an HA-tagged HDAC1 cDNA were co-transfected into Cos-7 cells to determine effects of combinations of VQRKRQK mutations on the interaction (Fig 2D). Intriguingly, sequential mutations of the V359, R361 and K362 residues up to R363 resulted in an apparent increase in HDAC1 interaction as determined by Co-IP, however a construct ( $\mathrm{p} 50^{\mathrm{Mu}}$ ) that combines these sequential mutations with mutation of K365 resulted in an almost complete loss of HDAC1:p50 binding in the Co-IP (Fig 2D). Since independent mutation of K365 was insufficient to disrupt the HDAC1:p50 interaction (Supp. 2A), we conclude that multiple amino acid contacts are required for association with the VQRKRQK motif. Transfection of WT p50FLAG and $\mathrm{p} 50^{\mathrm{Mu}}$-FLAG constructs into human HeLa cells confirmed the ability of p50-FLAG to Co-IP endogenous HDAC1 and this experiment also validated in $n f k b 1^{-/}$MEFs (Supp. 2B) that disruption of the VQRKRQK motif in $\mathrm{p} 50^{\mathrm{Mu}}$ prevents the HDAC1 interaction (Fig 2E). Taken together these data provide evidence for sequence specificity of interaction of HDAC1 with the c-terminal end of p50 and suggest that multiple amino acids within the previously identified NLS are required to form a p50:HDAC1 complex. The p50 NLS is proposed to have a helical-like structure but with a high degree of flexibility that may enable interaction with several distinct molecular targets such as members of the IкB family as well as the nuclear importin receptors [20]. 

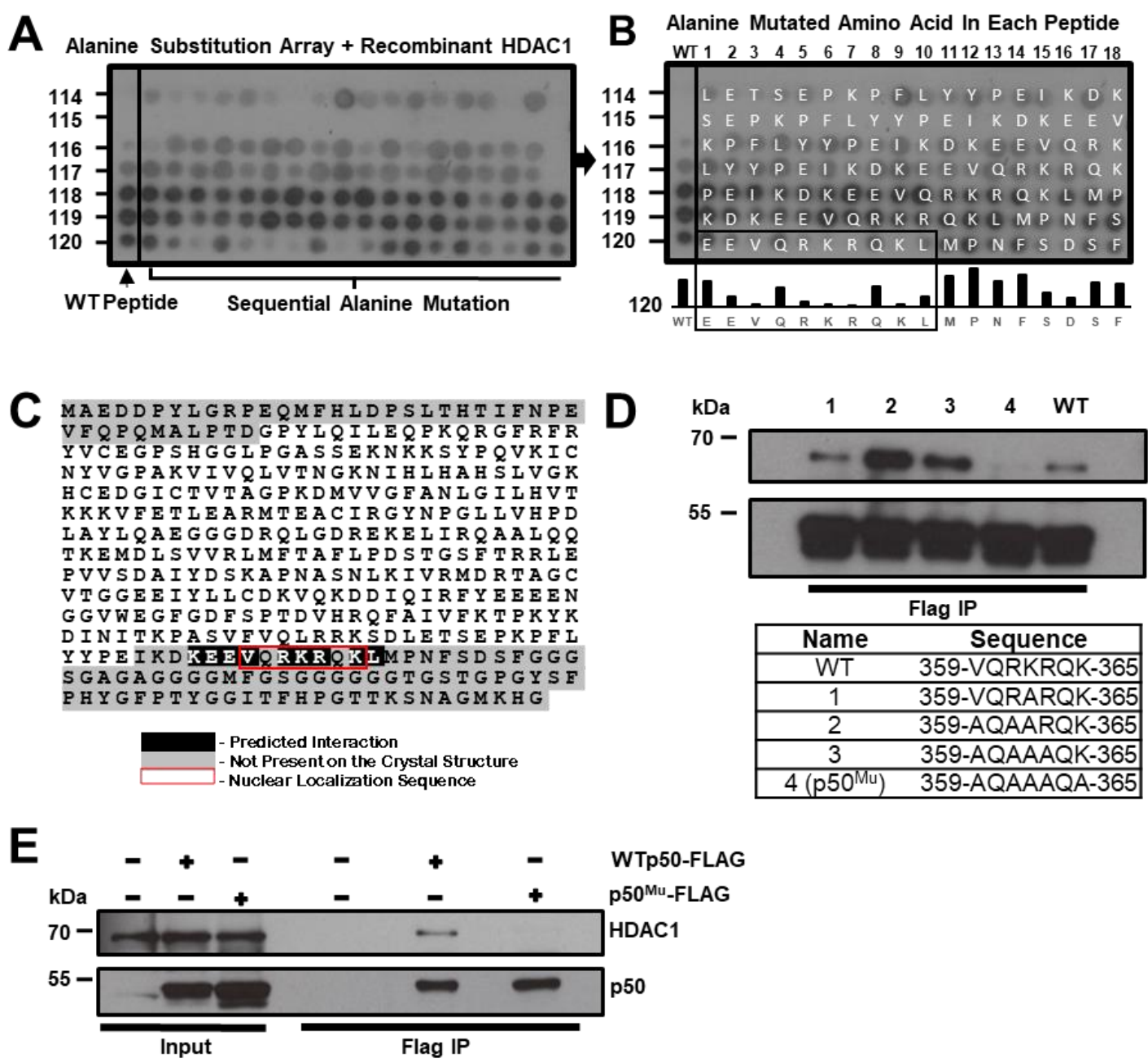

Figure 2. HDAC1 interaction is mediated by residues near the p50 nuclear localization sequence

A. Alanine substitution peptide array of the terminal seven 18aa peptides probed with recombinant HDACl displaying differential binding based on specific amino acid changes (anti-6x-His Tag Monoclonal Antibody HRP, R931-25, Invitrogen). B. Representation listing all of the specific amino acids mutated to alanine within each peptide $C$. Residues which attenuate the interaction between HDAC1 and p50 peptides in context of the full-length protein $D$. Western blot analysis of a FLAG immunoprecipitation assay of indicated mutant p50 expressed in Cos7 cells transiently transfected along with HDAC1-HA. Complete mutation of the indicated residues (Sequence 4) result in a near total ablation of the p50:HDAC1 interaction in full length transiently transfected proteins (anti p50-HRP [ab195854 Abcam] anti-HDAC1-HRP conjugate [ab193096 Abcam]). E. Western blot analysis of a FLAG coimmunoprecipitation assay of HELA cells transiently transfected with WT or p50Mu mutant confirming loss interaction with endogenous HDACl under resting conditions. Representative image of three independent experiments. Antibodies are those used in panel $D$. 


\subsection{Fundamental functions of p50 are unaffected by loss of the HDAC1 interaction motif.}

We were next interested in determining the functional impacts of p50 mutations that perturb HDAC1 binding. As shown in Fig 3A, binding of p65 and $\mathrm{I} \kappa \mathrm{Ba}$ was unaffected by mutations in the $\mathrm{p} 50^{\mathrm{Mu}}$ protein in U20S cells. Similarly there was no effect of mutation of the VQRKRQK motif on the I $\kappa \mathrm{B} \alpha$ interaction in $n f k b 1^{-}$murine embryonic fibroblasts (Fig 3B), although we cannot rule out that pull down of $\mathrm{I} \kappa \mathrm{B} \alpha$ occurred due to interaction with $\mathrm{p} 65$ in a p50 ${ }^{\mathrm{Mu}}: \mathrm{p} 65: \mathrm{I} \kappa \mathrm{B} \alpha$ complex. We also observed no differences in the ability of WT p50 and p50 ${ }^{\mathrm{Mu}}$ proteins to stimulate $\mathrm{NF}-\kappa \mathrm{B}-d e p e n d e n t$ transcription from co-transfected synthetic $(3 \mathrm{X} \kappa \mathrm{B}-\mathrm{Luc})$ and natural (IאB $\alpha$-Luc) promoters (Fig 3C). Hence, p50 $0^{\mathrm{Mu}}$ retains the ability to assemble p65:p50 heterodimers and to support classical canonical $\mathrm{NF}-\kappa \mathrm{B}$ signaling under the regulation of IкB $\alpha$. Perhaps more unexpected was the lack of effect of the VQRKRQK mutations on nuclear expression of $\mathrm{p} 50^{\mathrm{Mu}}$, verified by Western blotting in $n f k b 1^{-/}$MEFs and U20S cells (Fig 3D and Supp. 3A and B) and immunofluorescent localization (Fig 3E and Supp. 3C). Moreover, this nuclear localization occurs in both resting and TNFa stimulated MEF's independent of expressed protein concentration (Supp. 2C). Additionally, ChIP assays on exogenously expressed FLAGtagged proteins suggested that VQRKRQK mutations displayed slight but non-significant reduction of binding of p50 with well-known target promoters $c x c l 1$ and $c x c l 2$, again confirming the presence of $\mathrm{p} 0^{\mathrm{Mu}}$ in the nucleus of transformed cells (Fig 3F). These data therefore argue against an essential role of the NLS for p50 nuclear translocation and while being inconsistent with the original report that suggested an absolute requirement of the NLS, these data are in agreement with more recent published studies that experimentally demonstrate the NLS is probably non-essential for nuclear localization [10,21]. However, we did note that our immunofluorescence images suggested higher cytoplasmic expression for $\mathrm{p} 50^{\mathrm{Mu}}$ compared with WT (Figure 3E). This observation may indicate that the NLS influences the efficiency of nuclear translocation. A caveat to this suggestion is an apparent technical difference between the Western blotting and immunofluorescence for detection of cytoplasmic p50, with the former we were able to detect similar abundant levels of cytoplasmic WT and mutant p50 proteins while with the latter we failed to observe WT p50 in the cytoplasm. Since our controls for nuclear contamination of cytoplasmic protein extracts were clean we are confident that the Western blot 
is detecting cytoplasmic p50. A possible explanation for the differences between the protocols is that in the native state of the immunofluorescence experiments, the FLAG epitope may be somehow masked in the cytoplasm. Further, since the FLAG tag is fused to the c-terminal end of $\mathrm{p} 50$, mutations in this region in the $\mathrm{p} 50^{\mathrm{Mu}}$ protein may have unmasked the FLAG epitope and explain why the mutant and not WT is detected. Despite these technical caveats, collectively the data show that the mutations in the NLS aimed at disruption of HDAC1 binding do not have a major impact on fundamental functions of p50 including its nuclear translocation or its ability to interact with target $\mathrm{\kappa B}$ DNA binding sites. 
A

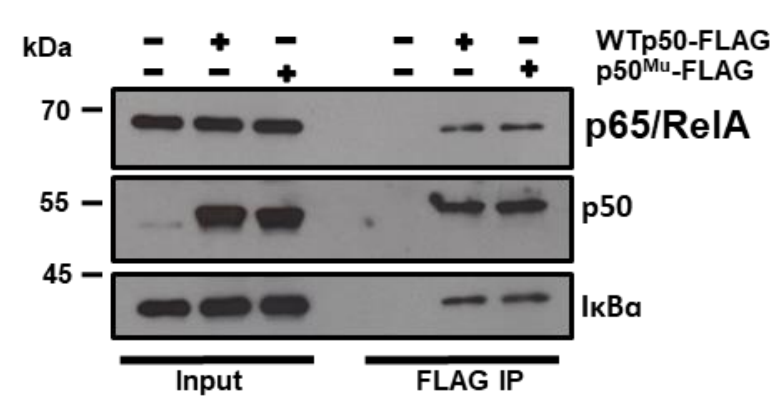

B

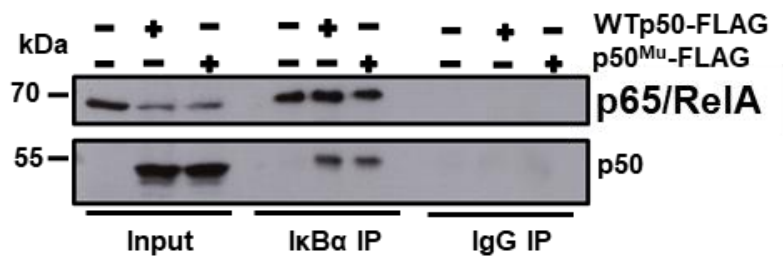

TNFa -

TNFa +
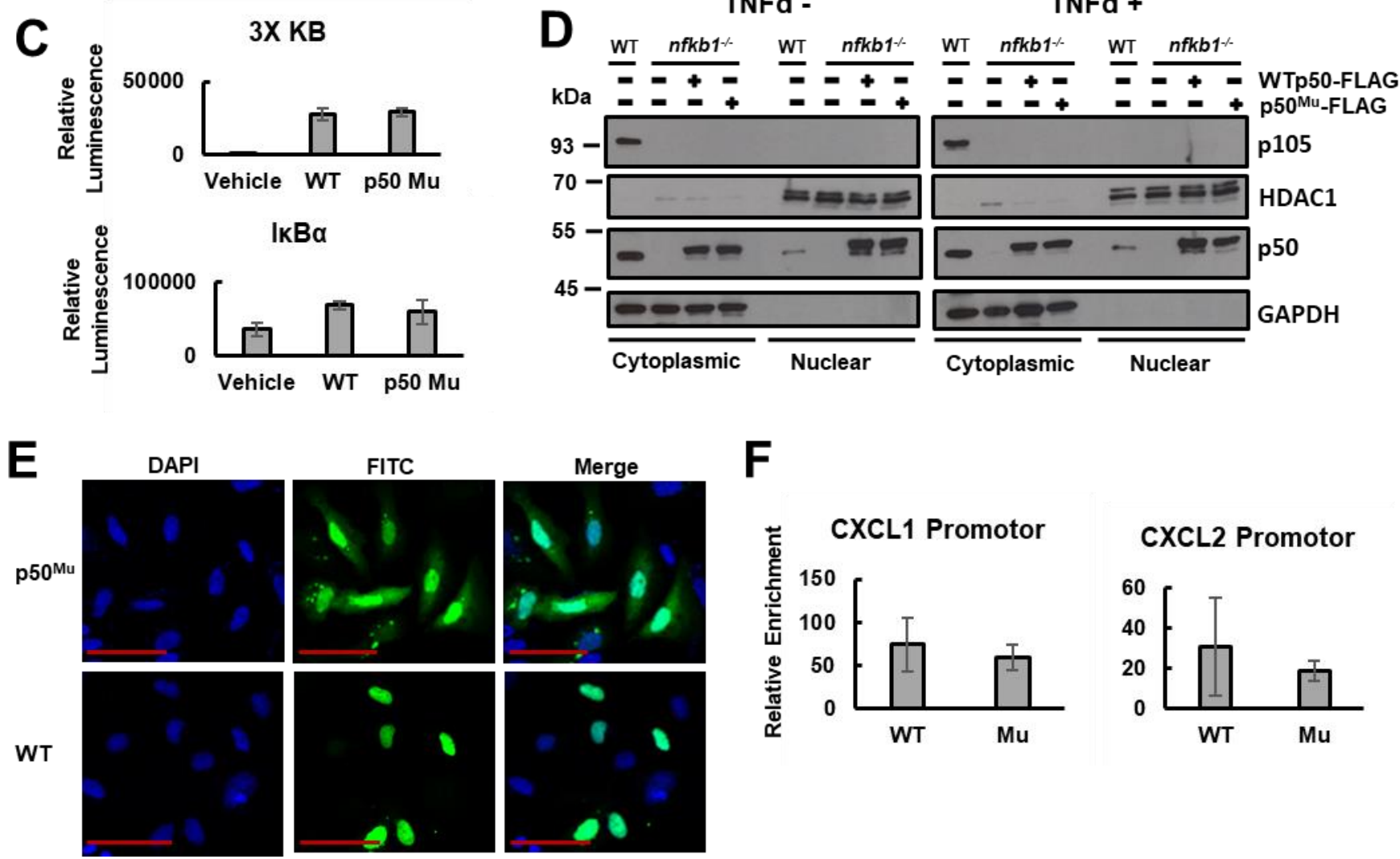

WT

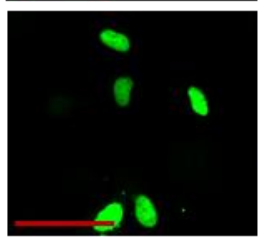

Figure 3. Canonical interactions of p50 are unaffected by the HDAC1 interruption mutation

A. FLAG immunoprecipitation of transiently expressed p50 in U2OS cells confirming similar coprecipitation of the endogenous partner p65 and I $\kappa$ Ba. B. Western blot co-immunoprecipitation of transfected p50 and endogenous p65 with endogenous I $\kappa \alpha$ pulldown confirming integrity of the canonical cytoplasmic inhibitory complex is unaffected by the p50Mu mutant in nfkb1 ${ }^{-1}$ MEFs. $C$. Luciferase reporter assay for a $3 \times \mathrm{NF}-\kappa B$ promotor and I $\kappa B \alpha$ promotor in $n f k b 1^{-/}$MEFs reconstituted with WT or p50Mu confirming similar ability to promote $N F-\kappa B$ dependent gene transcription. D. Western blot of nuclear and cytoplasmic extracts from WT and transiently transfected nfkb1 ${ }^{-1}$ MEFs $(200 \mathrm{ng} / \mathrm{ml})$ showing 550 nuclear translocation is unaffected by the mutation despite its proximity to and inclusion of a portion of the nuclear localization sequence $\boldsymbol{E}$. Immunofluorescence confocal microscopy of U2OS cells transiently transfected with WT-FLAG or p50Mu-FLAG detected by anti-FLAG-FITC

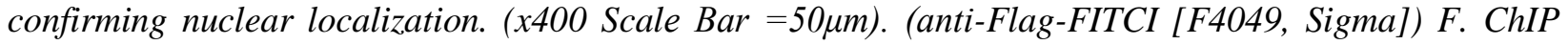
assay for p50 in $\mathrm{fkbl}^{--} \mathrm{MEFs}$ confirming similar binding at Cxcl1 and Cxcl2 promotors. All western blots are representative images of three independent experiments: anti-p105/p50-HRP (ab195854 Abcam), anti-HDAC1-HRP (ab193096 Abcam), anti-p65 (sc372 Santa Cruz), anti-GAPDH (ab22555 Abcam). 


\subsection{HDAC1:p50 regulates chromatin remodeling at neutrophil chemokine genes.}

Having established that disruption of the HDAC1 interaction motif did not affect p50 binding to $\mathrm{\kappa B}$ sites in the $\mathrm{Cxcll}$ and $\mathrm{Cxcl} 2$ genes we next determined if there was an effect on local chromatin structure in the promoter regions of these genes. To this end, we performed ChIP assays using a pan-acetylated lysine Histone 3 (Ac-H3) antibody on chromatin isolated from $n f k b 1^{-1}$ MEFs expressing either WT p50-FLAG or $\mathrm{p} 50^{\mathrm{Mu}}$-FLAG proteins (Supp. 4A). As shown in Fig 4A, histone lysine acetylation density was enriched at the cxcll and cxcl2 promoters of cells expressing $\mathrm{p} 50^{\mathrm{Mu}}$ relative to cells expressing the WT protein. This effect reached statistical significance for $\mathrm{cxcl}$, whereas a trend for enriched Ac-H3 was observed for Cxcll. Hence, mutations in the p50 NLS that disrupt its interaction with HDAC1 results in a more transcriptionally active state of chromatin at promoters of genes encoding two key neutrophil chemokines. From these data we predicted that the expression of $\mathrm{Cxcll}$ and $\mathrm{Cxcl} 2$ and that of other known p50-target genes would be enhanced in cells expressing $\mathrm{p} 50^{\mathrm{Mu}}$. $n f k b 1^{-/}$MEFs were therefore transfected with WT p50 or $\mathrm{p}^{\mathrm{Mu}}$ (Fig 4B) prior to stimulation with $\mathrm{TNF} \alpha$ and quantification of chemokine and cytokine gene expression. Quantification of mRNAs for Cxcll and $\mathrm{Cxcl} 2$ revealed that $\mathrm{TNF} \alpha$ stimulation was higher for both transcripts in the presence of $\mathrm{p} 50^{\mathrm{Mu}}$ (Fig 4C). In addition, following TNF $\alpha$ stimulation transcripts for Il-6, CxcllO and Mmpl3 were also increased to a greater degree in the $\mathrm{p} 50^{\mathrm{Mu}}$ transfected MEFs (Fig 4C). Moreover, ELISA for IL-6 in culture media confirmed that $\mathrm{p} 50^{\mathrm{Mu}}$ transfected cells secreted higher levels of the cytokine in comparison to the WT transfectants (Fig 4D). Cells used in these experiments displayed a transfection efficacy of $73.7 \%$ (Supp. 4B). Finally, neutrophil migration assays revealed that conditioned media from TNF-stimulated $\mathrm{p} 50^{\mathrm{Mu}}$ expressing cells (Supp. 4C) resulted in enhanced neutrophil chemotaxis compared with media from cells expressing WT p50 (Fig 5). We conclude that the p50-HDAC1 interaction restricts neutrophil chemoattraction under conditions of inflammatory stimulation. 

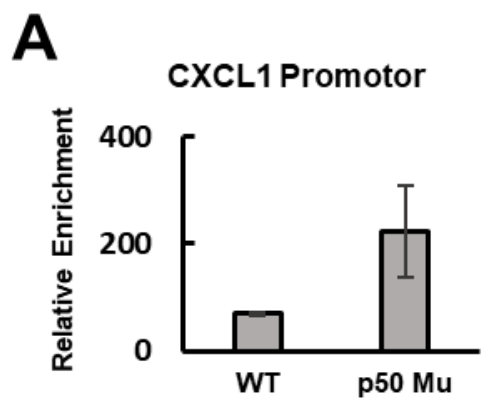

C

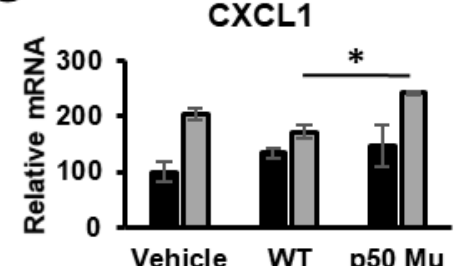

CXCL10

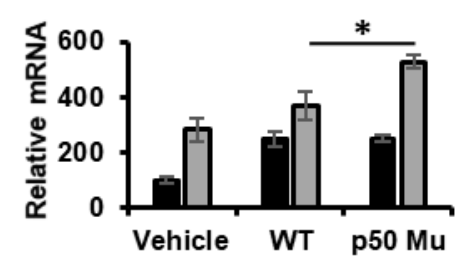

IL-6

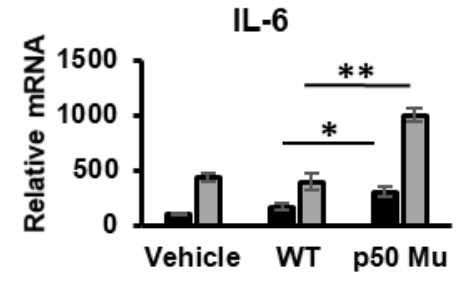

CXCL2 Promotor

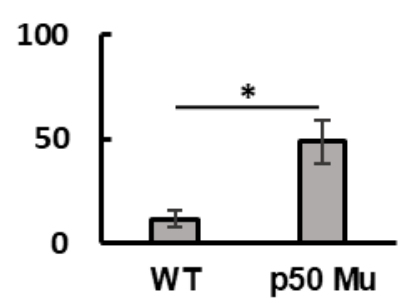

CXCL2

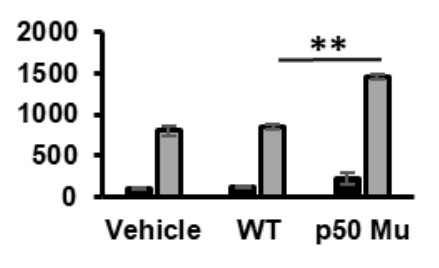

MMP13

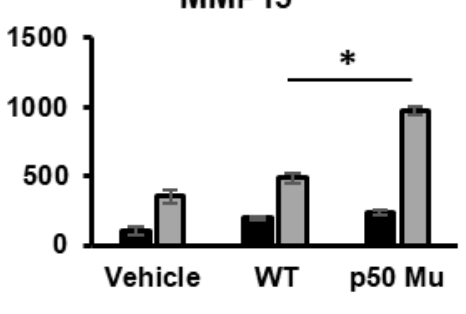

B
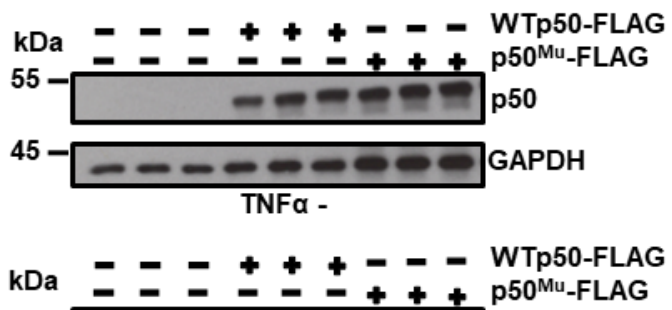

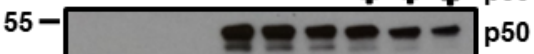

$45-(---\cdots-\cdots$ GAPDH

TNFa+

D IL-6 ELISA

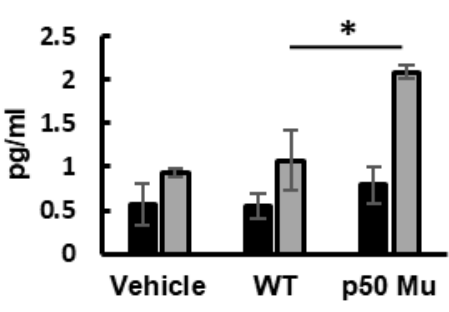

TNFa- QTNFa+

TNF $\alpha$ - 口TNF $\alpha+$

Figure 4. Loss of the p50:HDAC1 interaction leads to an increased pro-inflammatory profile

A. Pan acetyl Histone 3 ChIP assay qRT-PCR demonstrating a significant increase in acetylation of the well-known p50 target gene Cxcl2 when $n f k b 1^{--}$MEFs are transfected with mutant p50 unable to bind HDACl ( $p 50 M u)$ compared to WT $(p=0.021)$. The same trend is observed in the target gene Cxcll. B. Western blot analysis confirming equal transient transfection of WT and p50Mu in $n f k b 1^{-1-}$ MEFs untreated or treated with TNFa (n=3) antibodies: anti-p50-HRP (ab195854 Abcam), anti-GAPDH (ab22555 Abcam). C. RT-qPCR demonstrating a significant increase in mRNA expression of well-known p50 repressed genes in the absence of HDACl interaction (p50Mu) in nfkb1 ${ }^{-1-}$ MEFs. Il-6 p=0.002, Cxcl2 $p=0.0002$, Cxcl1 p=0.004, Cxcl10 $p=0.048$ and Mmpl3 $p=0.0004(n=3)$. D. ELISA analysis of cell media showing a significant increase in released IL-6 in nfkb1 ${ }^{-1}$ MEFs stimulated with TNFa or unstimulated $(p=0.047)(n=3)$. 


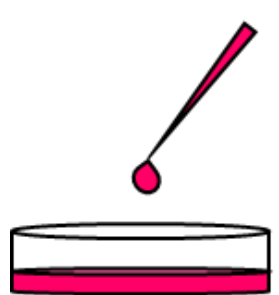

Transfect

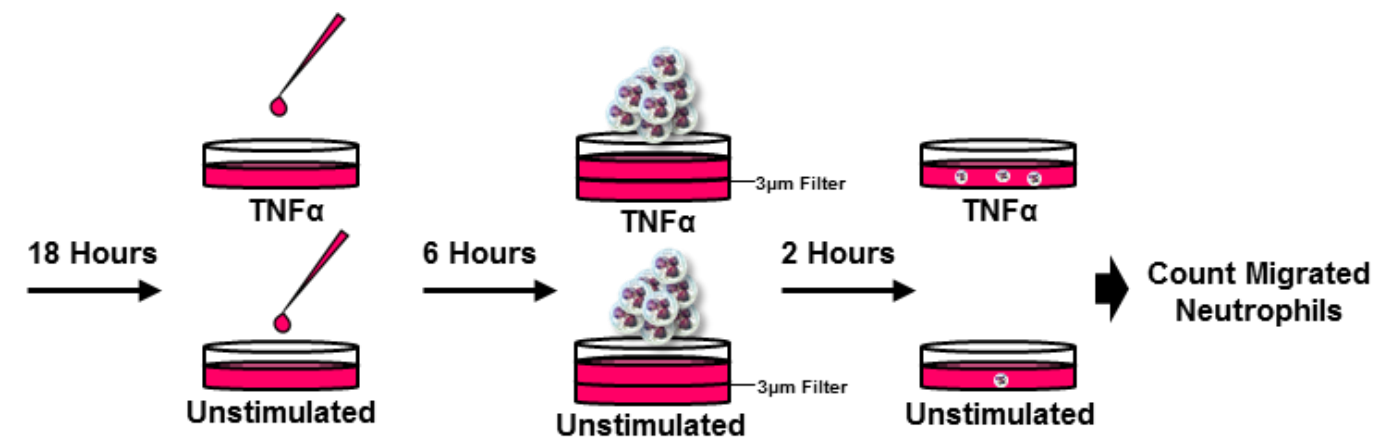

Neutrophil Chemotaxis

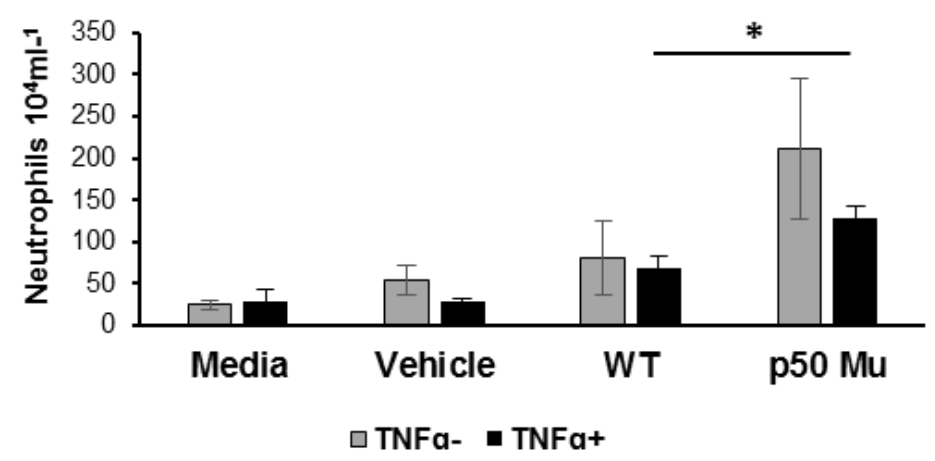

Figure 5. p50:HDAC1 represses neutrophil chemotaxis

Schematic of a neutrophil chemotaxis highlighting timeframes and cell counts post chemotaxis of murine neutrophils showing increased chemotaxis toward p50Mu conditioned media and significantly more infiltration when $\mathrm{fkbl}^{-/}$MEFs reconstituted with WT or p50Mu are stimulated with TNFa post transfection $(p=0.046)(n=3)$. 


\section{Conclusion}

In summary, we present combined in silico, in vitro and in vivo data that supports a hypothesis that direct interaction of HDAC1 with p50 homodimers bound at $\kappa \mathrm{B}$ sites of promoters and enhancers constitutes a key mechanism constricting NF- $\kappa \mathrm{B}-$ dependent transcription of at least a subset of inflammatory genes. The NLS is now emerging as an important nexus for interaction of p50 with a number of different regulatory proteins; HDAC1 now joining $\mathrm{Bcl} 3$ and $\mathrm{I} \kappa \mathrm{B} \alpha$ as identified NLS interacting factors. It is yet to be determined if these interactions are mutually exclusive. Bcl3 and HDAC1 seem to share at least the VQRKRQK motif which is required for each factor to complex with p50 but whether or not these are exclusive binding events warrants further investigation. As $\mathrm{Bcl} 3$ and $\mathrm{HDAC} 1$ provide distinct mechanisms through which p50 negatively controls gene expression, they may provide context-specific regulation that is determined by cell type, distinct $\kappa \mathrm{B}$ promoter/enhancer sequences, the nature of inflammatory signaling, influences of adjacent transcription factors or of other cofactors required for assembly of $\mathrm{Bcl} 3$ and HDAC1 repression complexes. As we observed an enrichment of lysine acetylation at $\mathrm{Cxcll}$ and $\mathrm{Cxcl} 2$ promoters when they were associated with $\mathrm{p} 50^{\mathrm{Mu}}$ we suggest that this reflects disruption of an HDAC-mediated rather than Bcl3-regulated activity. As such, we propose that the controlled assembly of HDAC:p50:p50 complexes at neutrophil chemokine genes is a physiological requirement for effective control of neutrophilic inflammation. 


\section{Acknowledgements}

The authors would like to thank their colleagues in the NFRG for their technical assistance and Prof. Neil Perkins for the provision of WT p50-FLAG pcDNA3.1 constructs. This work was supported by grants received from the Irish Cancer Society Grant (CRS12DOW), Science Foundation Ireland Grant (12/RI/2345) [P.K]. This work was also funded by Cancer Research UK Grant (C18342/A2390) and Medical Research Council Grant (MR/K001949) [D.A.M.].

\section{Author Contributions}

T.C. performed most of the experiments aided in experimental design and wrote the paper. J.W. and L.M. performed additional experiments and assisted with Chemotaxis and Immunofluorescence experiments. C.D. and P.K synthesized and provided all peptide arrays. A.K. and J.M. Performed and analyzed ChIP experiments. D.M. and C.W. Designed experiments and provided input and oversite of the project as well as assisted in writing the paper.

\section{Conflict of Interest}

The authors declare no conflicts of interest. 


\section{References}

1. Cartwright T, Perkins ND, L. Wilson C (2016) NFKB1: a suppressor of inflammation, ageing and cancer. FEBS J 283: 1812-1822.

2. Perkins ND, Gilmore TD (2006) Good cop, bad cop: the different faces of NF-kappaB. Cell Death Differ 13: 759-772.

3. Wilson CL, Jurk D, Fullard N, Banks P, Page a, Luli S, Elsharkawy a M, Gieling RG, Chakraborty JB, Fox C, et al. (2015) NFkB1 is a suppressor of neutrophil-driven hepatocellular carcinoma. Nat Commun 6: 6818.

4. Wessells J, Baer M, Young H a., Claudio E, Brown K, Siebenlist U, Johnson PF (2004) BCL-3 and NF-kB p50 attenuate lipopolysaccharide-induced inflammatory responses in macrophages. $J$ Biol Chem 279: 49995-50003.

5. Lin L, Kobayashi M (2003) Stability of the Rel homology domain is critical for generation of NFkappa B p50 subunit. J Biol Chem 278: 31479-31485.

6. Kravtsova-Ivantsiv Y, Shomer I, Cohen-Kaplan V, Snijder B, Superti-Furga G, Gonen H, Sommer T, Ziv T, Admon A, Naroditsky I, et al. (2015) KPC1-Mediated Ubiquitination and Proteasomal Processing of NF-кB1 p105 to p50 Restricts Tumor Growth. Cell 161: 333-347.

7. Hou S, Guan H, Ricciardi RP (2003) Phosphorylation of serine 337 of NF-kappaB p50 is critical for DNA binding. J Biol Chem 278: 45994-45998.

8. Sha WC, Liou HC, Tuomanen EI, Baltimore D (1995) Targeted disruption of the p50 subunit of NF-kappa B leads to multifocal defects in immune responses. Cell 80: 321-330.

9. Elsharkawy AM, Oakley F, Lin F, Packham G, Mann D a, Mann J (2010) The NF-kappaB p50:p50:HDAC-1 repressor complex orchestrates transcriptional inhibition of multiple proinflammatory genes. J Hepatol 53: 519-527.

10. Collins PE, Kiely P a., Carmody RJ (2014) Inhibition of transcription by B cell leukemia 3 (Bcl-3) protein requires interaction with nuclear factor $\kappa \mathrm{B}(\mathrm{NF}-\kappa \mathrm{B})$ p50. J Biol Chem 289: 7059-7067.

11. Zhong H, May MJ, Jimi E, Ghosh S (2002) The Phosphorylation Status of Nuclear NF-KB Determines Its Association with CBP/p300 or HDAC-1. Mol Cell 9: 625-636.

12. Kramer A, Schneider-Mergener J (1998) Synthesis and Screening of Peptide Libraries on 
Continuous Cellulose Membrane Supports BT - Combinatorial Peptide Library Protocols. In Cabilly S (ed.) pp 25-39. Humana Press, Totowa, NJ.

13. Ghosh G, Duyne G Van, Ghosh S, Sigler PB (1995) Structure of NF-[kappa]B p50 homodimer bound to a [kappa]B site. Nature 373: 303-310.

14. Millard CJ, Watson PJ, Celardo I, Gordiyenko Y, Cowley SM, Robinson C V., Fairall L, Schwabe JWR (2013) Class I HDACs share a common mechanism of regulation by inositol phosphates. Mol Cell 51: 57-67.

15. Pettersen EF, Goddard TD, Huang CC, Couch GS, Greenblatt DM, Meng EC, Ferrin TE (2004) UCSF Chimera -A visualization system for exploratory research and analysis. J Comput Chem 25: $1605-1612$.

16. Zhong H, May MJ, Jimi E, Ghosh S (2002) The Phosphorylation Status of Nuclear NF-kB Determines Its Association with CBP/p300 or HDAC-1. Mol Cell 9: 625-636.

17. Tovchigrechko A VI (2006) GRAMM-X public web server for protein-protein docking. Nucleic Acids Res 34: 310-304.

18. Malek S, Huxford T, Ghosh G (1998) Ikappa Balpha Functions through Direct Contacts with the Nuclear Localization Signals and the DNA Binding Sequences of NF-kappa B. J Biol Chem 273: $25427-25435$.

19. Collins PE, Grassia G, Colleran A, Kiely P a., Ialenti A, Maffia P, Carmody RJ (2015) Mapping the interaction of B cell leukaemia 3 (Bcl-3) and nuclear factor $\kappa \mathrm{B}(\mathrm{NF}-\kappa \mathrm{B}) \mathrm{p} 50$ identifies a Bcl-3mimetic anti-inflammatory peptide. J Biol Chem 3: jbc.M115.643700.

20. Fagerlund R, Kinnunen L, Köhler M, Julkunen I, Melén K (2005) NF-кB Is Transported into the Nucleus by Importin $\alpha 3$ and Importin $\alpha 4$. J Biol Chem 280: 15942-15951.

21. Blank V, Kourilsky P, Israël A (1991) Cytoplasmic retention, DNA binding and processing of the NF-kappa B p50 precursor are controlled by a small region in its C-terminus. EMBO J 10: 4159 4167. 


\section{Tables}

\begin{tabular}{|c|c|c|}
\hline \multicolumn{2}{|r|}{ Mutagenesis Primers } & $\mathrm{Tm}$ \\
\hline AQAAAQA Forward & agctcaggcgCTCATGCCCAATTTTTCG & 60 \\
\hline AQAAAQA Reverse & gccgcctgcgcTTCTTCTTTATCTTTGATTTCAGG & 60 \\
\hline AQAAAQK Forward & agctcagaagCTCATGCCCAATTTTTCG & 60 \\
\hline AQAAAQK Reverse & gccgcctgcgcTTCTTCTTTATCTTTGATTTCAGG & 60 \\
\hline AQAARQK Forward & acgtcagaagCTCATGCCCAATTTTTCG & 60 \\
\hline AQAARQK Reverse & gccgcctgcgcTTCTTCTTTATCTTTGATTTCAGG & 60 \\
\hline VQAARQK Forward & acgtcagaagCTCATGCCCAATTTTTCG & 60 \\
\hline VQAARQK Reverse & gccgcctgcacTTCTTCTTTATCTTTGATTTCAGG & 60 \\
\hline VQRARQK Forward & acgtcagaagCTCATGCCCAATTTTTCG & 60 \\
\hline VQRARQK Reverse & gccctctgcacTTCTTCTTTATCTTTGATTTCAGG & 60 \\
\hline \multicolumn{3}{|c|}{ qRT-PCR Primers } \\
\hline mIL-6 Forward & GAGGATACCACTCCCAACAGA & 60 \\
\hline mlL-6 Reverse & AAGTGCATCATCGTTGTTCATA & 60 \\
\hline $\mathrm{mCXCL} 1$ Forward & CTGGGATTCACCTCAAGAACATC & 60 \\
\hline mCXCL1 Reverse & CAGGGTCAAGGCAAGCCTC & 60 \\
\hline mCXCL2 Forward & CCAACCACCAGGCTACAGG & 60 \\
\hline mCXCL2 Reverse & GCGTCACACTCAAGCTCTG & 60 \\
\hline mCXCL10 Forward & AAGTGCTGCCGTCATTTCT & 57 \\
\hline mCXCL10 Reverse & GTGGCAATGATCTCAACACG & 57 \\
\hline mMMP13 Forward & CTTCTTCTTGTTGAGCTGGACTC & 57 \\
\hline mMMP13 Reverse & CTGTGGAGGTCACTGTAGACT & 57 \\
\hline \multicolumn{3}{|c|}{ ChIP Primers } \\
\hline mCXCL1 Forward & GTTGGCAAAAGCAAACCACC & 57 \\
\hline mCXCL1 Reverse & ACTACAGTGATTTGCGGGGA & 57 \\
\hline mCXCL2 Forward & GACATCCCAGGGTCCCATAG & 57 \\
\hline mCXCL2 Reverse & TGCACGATGTCTGGAAAAGC & 57 \\
\hline
\end{tabular}

\section{Table 1}

Table of primers used for site directed mutagenesis, RT-qPCR and Chromatin immunoprecipitation PCR. 


\begin{tabular}{|c|c|c|c|}
\hline \multirow[b]{2}{*}{ Construct } & \multirow[b]{2}{*}{ Backbone } & \multicolumn{2}{|c|}{ Concentration } \\
\hline & & IP/Functional & Luciferase Assay \\
\hline WT-p50-FLAG & pcDNA3.1 & $200 \mathrm{ng} / \mathrm{ml}$ & $250 \mathrm{ng} / \mathrm{ml}$ \\
\hline VQRARQK- p50-FLAG & pcDNA3.1 & $200 \mathrm{ng} / \mathrm{ml}$ & $250 \mathrm{ng} / \mathrm{ml}$ \\
\hline AQAARQK-p50-FLAG & pcDNA3.1 & $200 \mathrm{ng} / \mathrm{ml}$ & $250 \mathrm{ng} / \mathrm{ml}$ \\
\hline AQAAAQK-p50-FLAG & pcDNA3.1 & $200 \mathrm{ng} / \mathrm{ml}$ & $250 \mathrm{ng} / \mathrm{ml}$ \\
\hline AQAAAQA-p50-FLAG & pcDNA3.1 & $200 \mathrm{ng} / \mathrm{ml}$ & $250 \mathrm{ng} / \mathrm{ml}$ \\
\hline 3x кB-Luc Reporter & pGL-2 & $\mathrm{N} / \mathrm{A}$ & $250 \mathrm{ng} / \mathrm{ml}$ \\
\hline CTL-Luc Reporter & pGL-2 & $\mathrm{N} / \mathrm{A}$ & $250 \mathrm{ng} / \mathrm{ml}$ \\
\hline I $\kappa \mathrm{B} \alpha$-Luc Reporter & pGL-2 & $\mathrm{N} / \mathrm{A}$ & $250 \mathrm{ng} / \mathrm{ml}$ \\
\hline Renilla- Luc-Reporter & pGL-2 & $\mathrm{N} / \mathrm{A}$ & $25 \mathrm{ng} / \mathrm{ml}$ \\
\hline
\end{tabular}

\section{Table 2}

Table of DNA plasmid constructs and the amounts used in the indicated application. 


\section{Supplemental Figures}

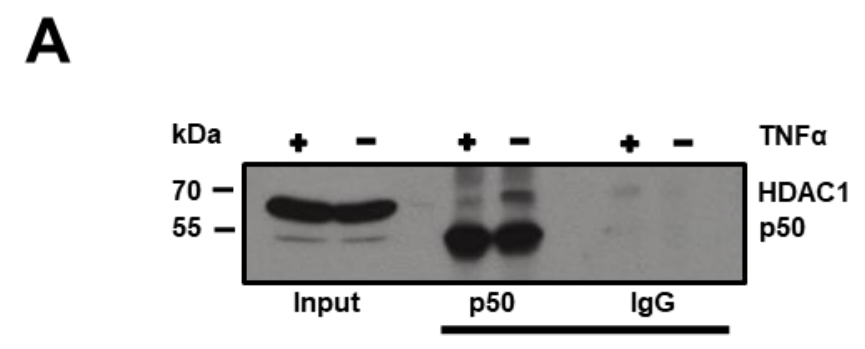

IP
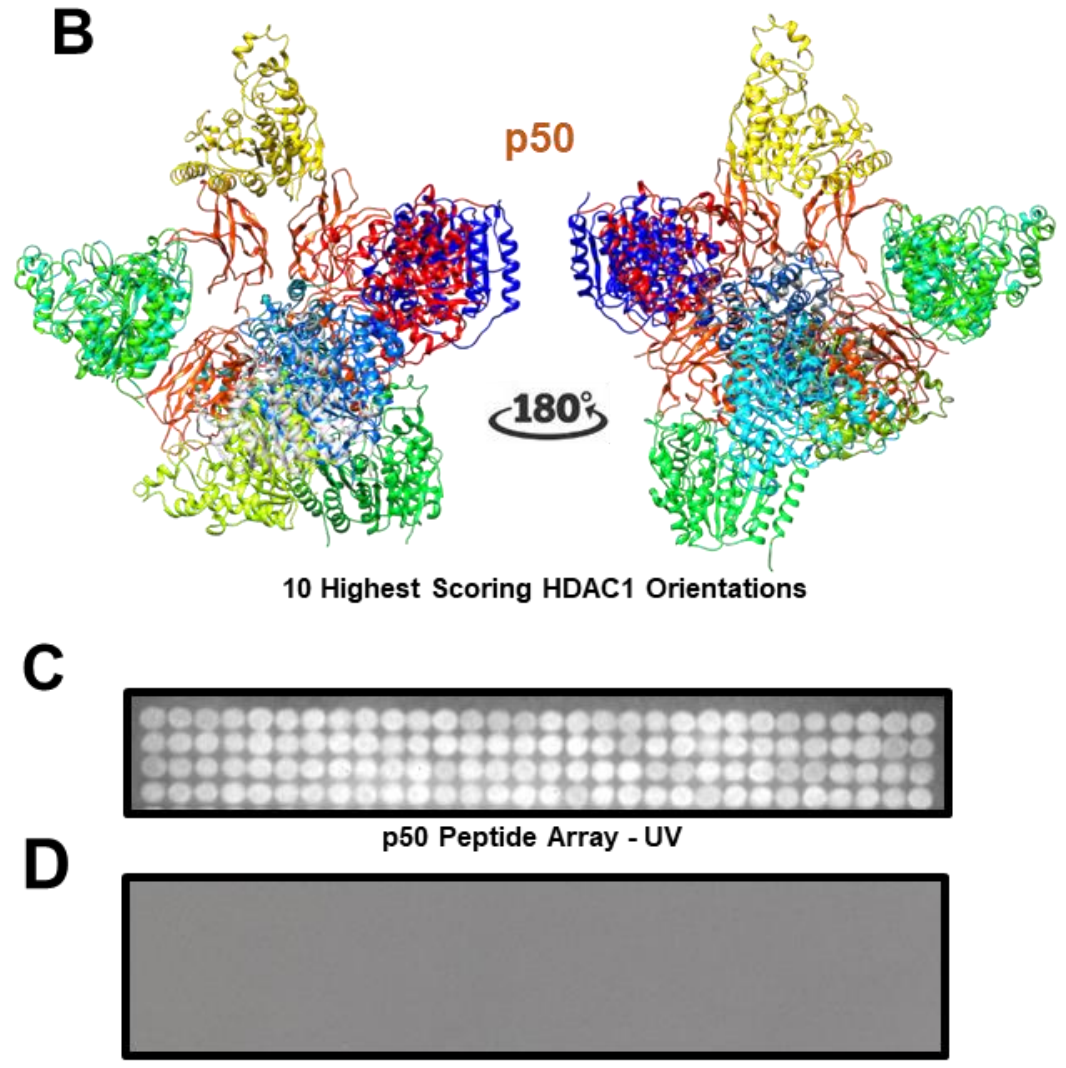

p50 Peptide Array - Anti 6X Ab

\section{Supplemental Figure 1}

A. Endogenous co-immunoprecipitation western blot of HELA cells which were untreated or treated with TNFa showing a reduction of HDAC1 interaction when stimulated (anti-p50-HRP (ab195854 Abcam), anti-HDAC1-HRP (ab193096 Abcam). B. Top ten highest scoring possible binding orientations between the p50 homodimer and HDAC1 crystal structures as predicted by the GRAMM$X$ server for rigid body interactions. $C$. p50 peptide array under UV light verifying the presence of 120 individual peptides spanning the length of p50. D. p50 peptide array probed with Anti-His $3 X-$ HRP antibody alone showing a lack of non-specific binding derived from the antibody used. 

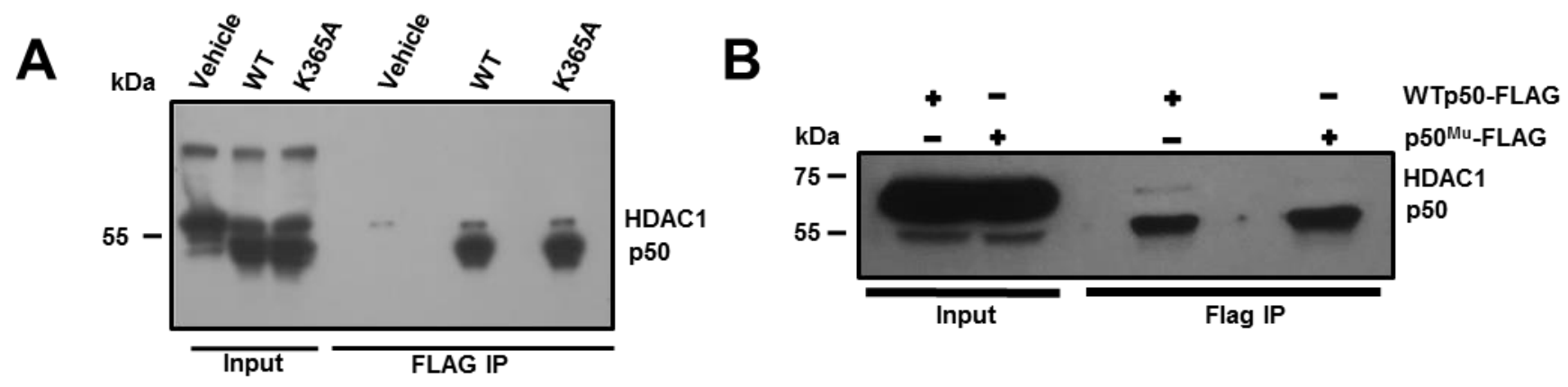

C

TNFa -

TNFa +

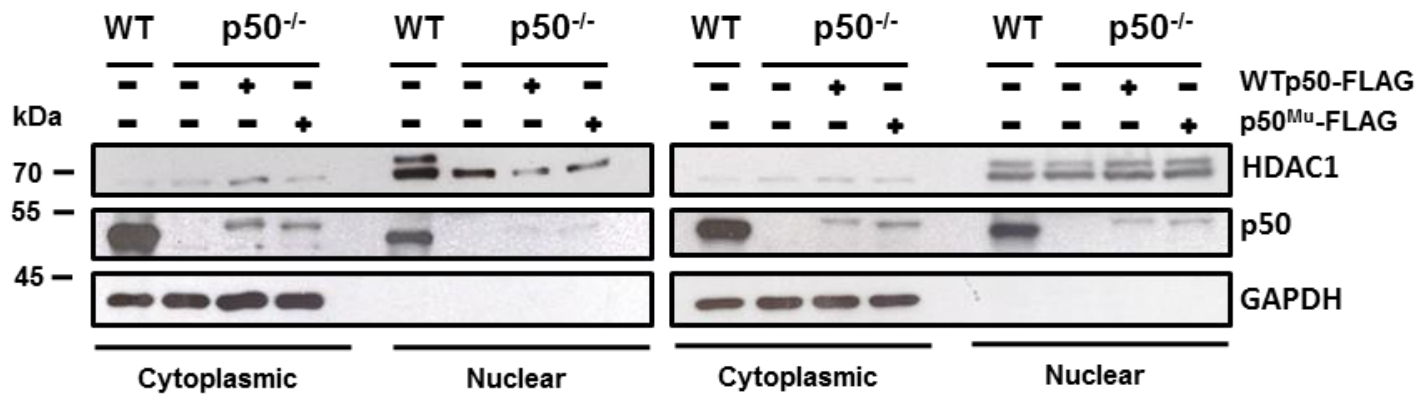

\section{Supplemental Figure 2}

A. Western blot analysis of a FLAG Co-IP assay showing similar binding of HDAC1 to WT or p50 ${ }^{K 365 A}$ mutant in Cos 7 cells. B. Western blot analysis of a FLAG Co-IP assay of nfkb1 $1^{-1-}$ MEFs showing a loss of interaction with endogenous HDACl in p50Mu Compared to WT. C. Western blot of nuclear and cytoplasmic extracts from WT and transiently transfected nfkb ${ }^{-1-}$ MEFs $(50 \mathrm{ng} / \mathrm{ml}$ of WT or $p 50^{M u}$ ) showing p50 nuclear translocation is unaffected by the mutation required to disrupt HDAC1 interaction despite its proximity to and inclusion of a portion of the nuclear localization sequence at reduced transient expression levels. [Detection antibodies anti-p105/p50-HRP (ab195854 Abcam), anti-HDAC1-HRP (ab193096 Abcam), and, anti-GAPDH (ab22555 Abcam). 

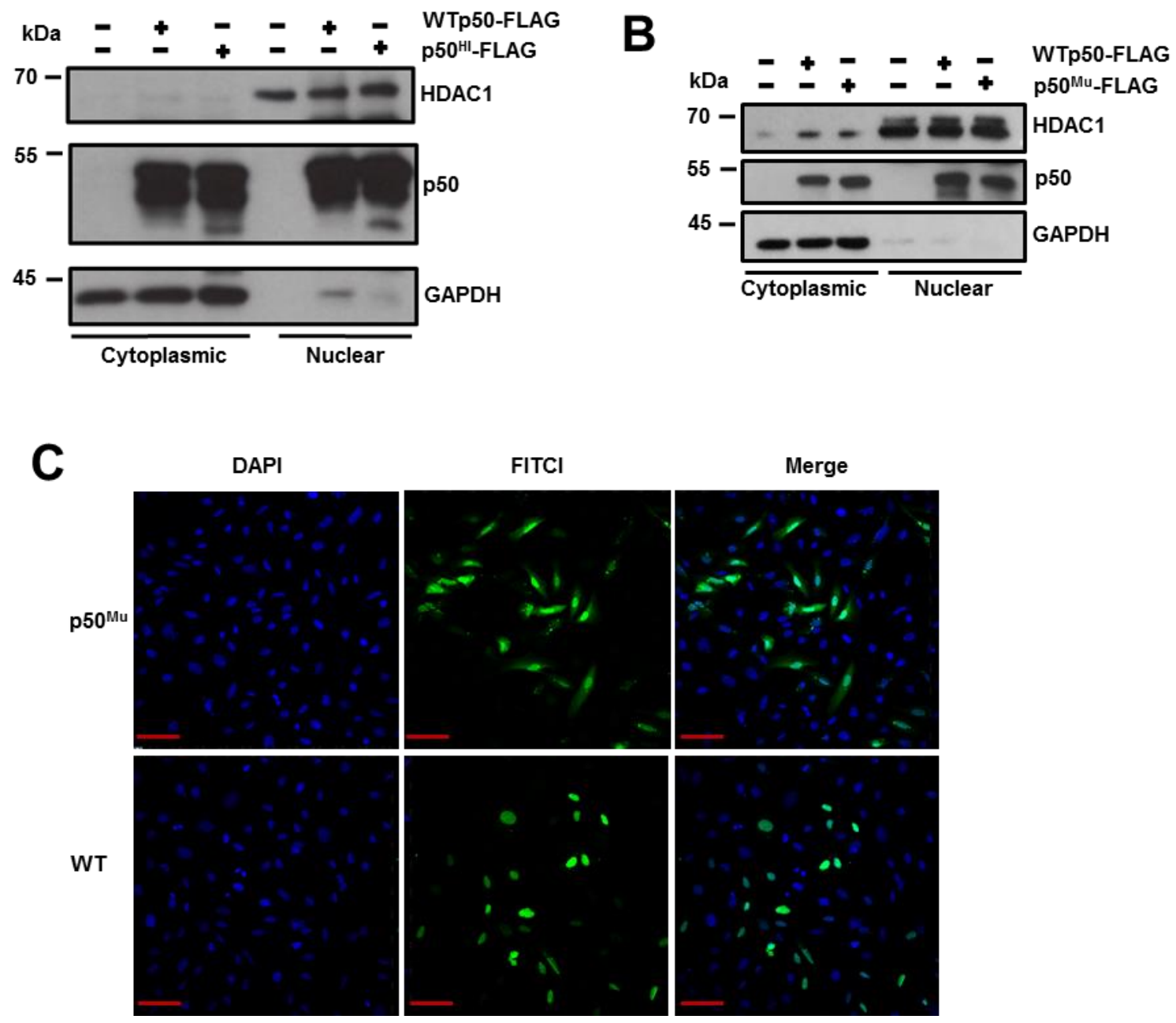

\section{Supplemental Figure 3}

A. Western blot of nuclear and cytoplasmic extracts from transiently transfected U2OS cells showing p50 nuclear translocation is unaffected by the mutation required to disrupt HDAC1 interaction despite its proximity to and inclusion of a portion of the nuclear localization sequence [anti-p50-HRP (ab195854 Abcam), anti-HDACl-HRP (ab193096 Abcam)] and anti-GAPDH (ab22555 Abcam). B. Western blot of nuclear and cytoplasmic extracts from transiently transfected nfkb1 ${ }^{-1} \mathrm{MEFs}$ $(200 \mathrm{ng} / \mathrm{ml})$ showing p50 nuclear translocation is unaffected by the mutation required to disrupt HDAC1 interaction despite its proximity to and inclusion of a portion of the nuclear localization sequence $\boldsymbol{C}$. Immunofluorescence confocal microscopy of U2OS cells transiently transfected with WTFLAG or p50Mu-FLAG detected by anti-FLAG-FITC confirming nuclear localization. (x200 Scale Bar $=50 \mu \mathrm{m})$. (anti-Flag-FITCI [F4049, Sigma]). 

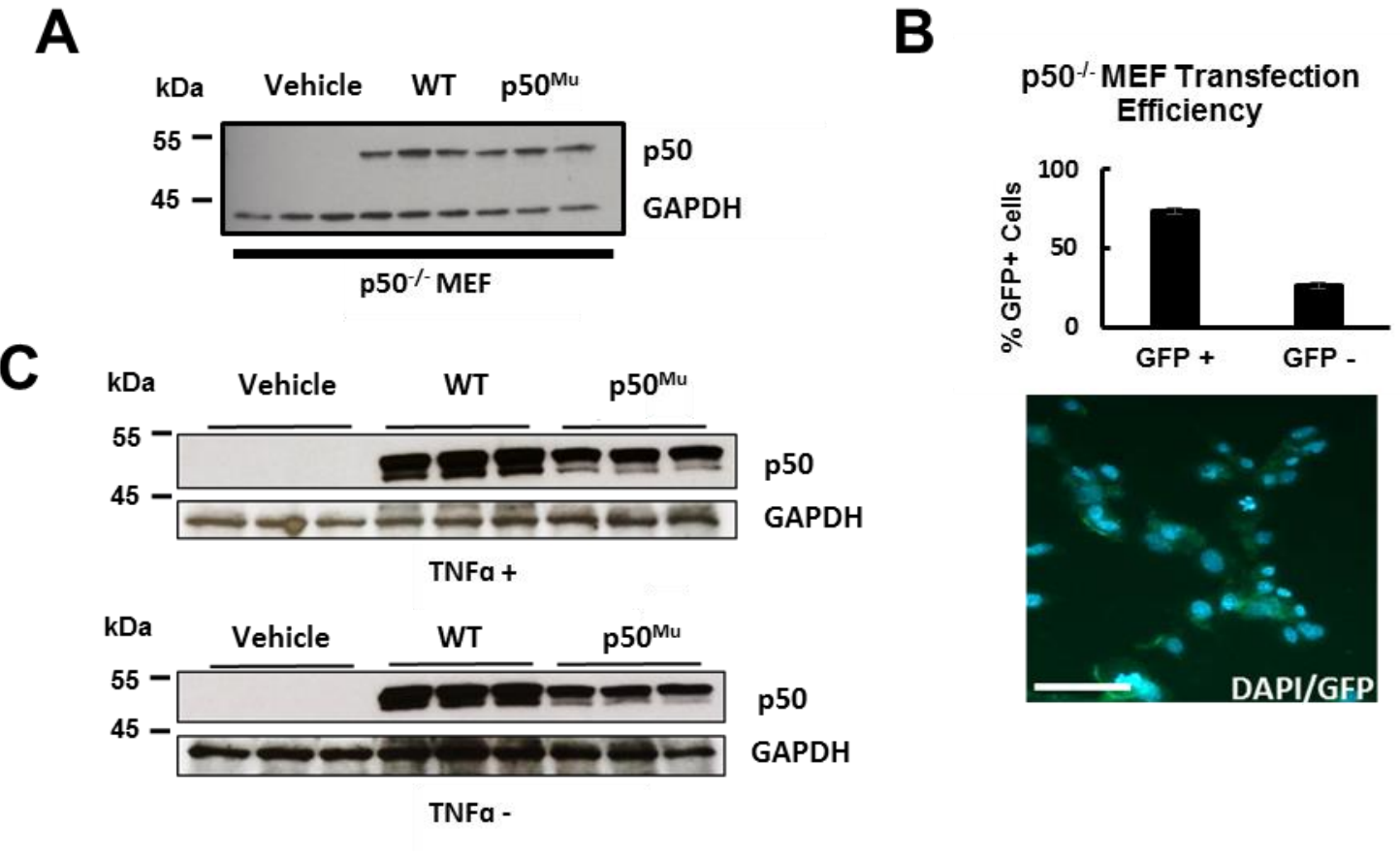

\section{Supplemental Figure 4}

A. Western blot showing comparable transfection of WT and p50Mu from $n f k b 1^{-1-}$ MEFs whose chromatin was used in pan $\mathrm{H} 3$ acetylation ChIP experiments. B. Transfection efficiency of $n f k b 1^{-/-}$ MEFs transfected with $200 \mathrm{ng} / \mathrm{ml} \mathrm{GFP}$ reporter vector $(73.7 \%$ xl00 Scale Bar=50um)). C. Western blot showing comparable transfection of WT and p50Mu from nfkb $1^{-1-}$ MEFs whose supernatant was used in the neutrophil chemotaxis experiments in both the presence and absence of TNFa. 